\title{
General theory of quantum fingerprinting network
}

\author{
Ji-Qian Qin $\odot,{ }^{1}$ Jing-Tao Wang, ${ }^{1}$ Yun-Long Yu $\odot,{ }^{1}$ and Xiang-Bin Wang $\odot^{1,2,3,4, *}$ \\ ${ }^{1}$ State Key Laboratory of Low Dimensional Quantum Physics, Department of Physics, \\ Tsinghua University, Beijing 100084, China \\ ${ }^{2}$ Shanghai Branch, CAS Center for Excellence and Synergetic Innovation Center in Quantum Information and Quantum Physics, \\ University of Science and Technology of China, Shanghai 201315, China \\ ${ }^{3}$ Jinan Institute of Quantum Technology, SAICT, Jinan 250101, China \\ ${ }^{4}$ Shenzhen Institute for Quantum Science and Engineering, and Physics Department, Southern University of Science and Technology, \\ Shenzhen 518055, China
}

(Received 16 November 2020; revised 7 April 2021; accepted 14 June 2021; published 9 July 2021)

\begin{abstract}
The purpose of fingerprinting is to compare long messages with low communication complexity. Compared with its classical version, quantum fingerprinting can realize exponential reduction in communication complexity. Recently, multiparty quantum fingerprinting is studied to determine whether the messages from many parties are all the same. However, the complete relationships among them, including which ones are the same, are more general for future applications. Here, we propose the relationship state $\mathcal{G}$ to describe all possible relationships among $N$ messages. Based on this, we provide a general quantum fingerprinting network and then take the four-party quantum fingerprinting protocol as an example for detailed analysis. We take the full parameter optimization for the quantum fingerprinting protocol through asymmetric channel. The method of encoding more than one bit on each coherent state is used to further improve the performance of our protocol. Compared with other protocols, our protocol provides more relationships among different parties with only linear increase in communication complexity.
\end{abstract}

DOI: 10.1103/PhysRevResearch.3.033039

\section{INTRODUCTION}

The application of quantum mechanics in the field of communication brings benefits in many aspects, such as security and communication complexity. With respect to security, quantum key distribution (QKD) [1-7] is a representative example that can provide information-theoretic security for private communication, while the security of its classical counterpart is based on the assumptions of computational complexity. In terms of communication complexity $[8,9]$, which is the minimum amount of communication required among participants to complete a certain task, compared with its classical version $[10,11]$, quantum fingerprinting can exponentially reduce the amount of information required for the tasks. This is very useful in energy-saving communication $[8,12,13]$.

The purpose of the fingerprinting protocol is to answer whether two $n$-bit messages $x_{1}$ and $x_{2}$ are the same based on their fingerprints. In the simultaneous message passing model [14], there are two senders sending the fingerprints of each one's original messages to the remote referee who needs to determine whether $x_{1}=x_{2}$ with a small error probability $P_{e}$ upper-bounded by $\varepsilon$. Quantum fingerprinting can

\footnotetext{
*xbwang@mail.tsinghua.edu.cn

Published by the American Physical Society under the terms of the Creative Commons Attribution 4.0 International license. Further distribution of this work must maintain attribution to the author(s) and the published article's title, journal citation, and DOI.
}

significantly reduce the communication complexity required to complete the comparison, i.e., the communication complexity of the classical version is $O(\sqrt{n})$-bits [10,11], while that of the quantum version is only $O\left(\log _{2} n\right)$-qubits [12]. However, the original protocol requires high-dimensional entanglement which makes its implementation technically challenging [12]. Fortunately, a quantum fingerprinting protocol based on coherent states is proposed [15] and since then, there have been many advances in theory and experiment [16-18]. Recently, to reduce the communication time, wavelength division multiplexing (WDM) is used in quantum fingerprinting and the result shows that not only the communication time is reduced, but also the communication complexity is further reduced [19].

So far, most studies have focused on two-party quantum fingerprinting protocols $[15-17,19]$ and there are only two relationships between the two inputs, $x_{1}=x_{2}$ or $x_{1} \neq x_{2}$. As the number of messages increases, things get more complicated and interesting. If we need to determine whether $N$ messages are all the same, then a simple way is to use the two-party quantum fingerprinting to compare $N$ inputs in pairs, which needs to be done $(N-1)$ times. So is there a more efficient way? Recently, a multiparty quantum fingerprinting is proposed [20], which only needs to be run once to determine whether $N$ inputs are the same. Obviously, in this case, the quantum fingerprinting network presents a huge advantage. However, the relationships among $N$ inputs are in general more complicated than the case above and hence it is not enough just to know whether they are all the same. Usually, we also need to know more relationships or even complete 
relationships among these inputs. Therefore, it is of great interest to study the multiparty quantum fingerprinting network with more relationships.

In this work, based on the existing framework [15,20], we study the complete relationships by changing the combination of the interference beams. We propose the relationship state $\mathcal{G}$ to describe all possible relationships and provide a general quantum fingerprinting network by the ingenious design of our protocol. In particular, we take the four-party quantum fingerprinting protocol as an example for detailed analysis. The method of encoding more than one bit on each coherent state is used to further improve the performance of our protocol. Moreover, we take the full parameter optimization for the quantum fingerprinting protocol through asymmetric channel. Compared with other protocols, our protocol provides more relationships among different parties with similar communication complexity.

The rest of this paper is arranged as follows. In Sec. II, we present our $N$-party quantum fingerprinting protocol. In Sec. III, we show the details of our protocol through the special case of a four-party quantum fingerprinting network. In Sec. IV, we study the behavior of our protocol through asymmetric channels. After this, we show the advantages of our protocol and how to further improve its performance by the multibit encoding method in Sec. V. Finally, we present a brief summary in Sec. VI.

\section{N-PARTY QUANTUM FINGERPRINTING PROTOCOL}

\section{A. Relationship state}

In general, given $N$ messages, they are not necessarily all the same. We can put the same messages into the same group (set). If there are $\mathcal{J}$ groups, then we name each group as $G_{1}, G_{2}, \ldots, G_{\mathcal{J}}$ and, of course, $1 \leqslant \mathcal{J} \leqslant N$.

Relationship state. The set of all groups for those $N$ messages. We shall denote it by $\mathcal{G}=\left\{G_{1}, G_{2}, \ldots, G_{\mathcal{J}}\right\}$. Since $\mathcal{G}$ is a set, it is only determined by its elements rather than the positions of them. We shall always arrange the positions of groups by the rule $\left|G_{a}\right| \geqslant\left|G_{b}\right|$ if $a \leqslant b$.

For example, given $N=4, \quad x_{1}=x_{2}=x_{3} \neq x_{4}, \quad \mathcal{G}=$ $\left\{G_{1}, G_{2}\right\}$, where $G_{1}=\left\{x_{1}, x_{2}, x_{3}\right\}, G_{2}=\left\{x_{4}\right\}$.

In Refs. [20,21], the following two special relationship states are studied.

All-equality relationship state. A special case that all messages are the same and we denote it as $\mathcal{G}^{\mathrm{AE}}$,

$$
\mathcal{G}^{\mathrm{AE}}=\left\{G_{1}\right\}
$$

where $G_{1}=\left\{x_{1}, x_{2}, \ldots, x_{N}\right\}$.

Exists-equality relationship state. A special case that there is at least a pair of equal messages and we denote it as $\mathcal{G}^{\mathrm{EE}}$,

$$
\mathcal{G}^{\mathrm{EE}}=\left\{G_{1}, G_{2}, \ldots, G_{\mathcal{J}}\right\},
$$

where $\left|G_{1}\right| \geqslant 2$.

The referee's task in our protocol is to determine the relationship state $\mathcal{G}$ for $N$ messages, given their fingerprints. The communication complexity of the protocol is the less the better, given $\varepsilon$.

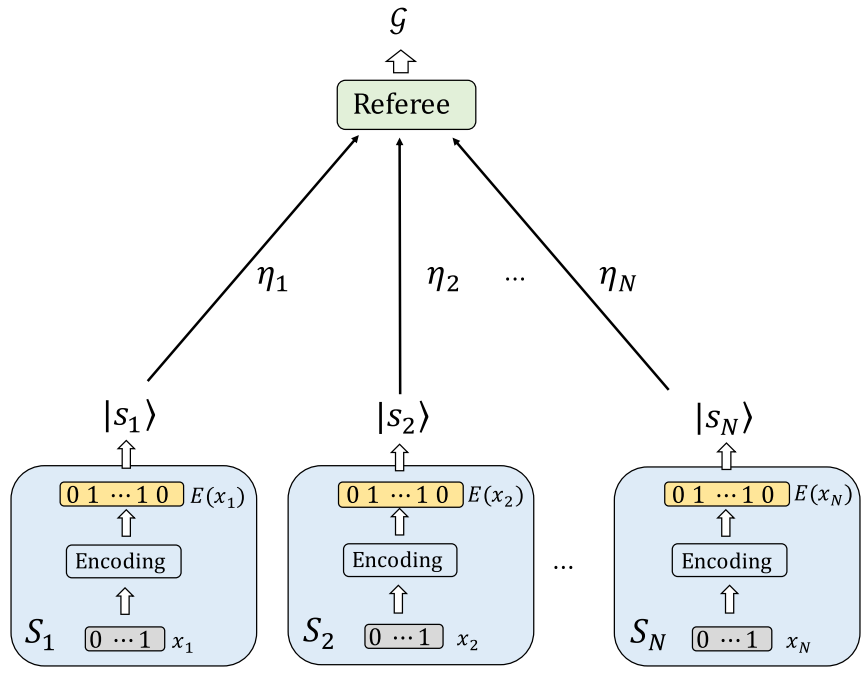

FIG. 1. $N$-party quantum fingerprinting protocol. There are $N$ senders $\left\{S_{k}\right\}, k=1,2, \ldots, N$. They encode the original messages $\left\{x_{k}\right\}$, shown in gray, with the ECC and change them into $\left\{E\left(x_{k}\right)\right\}$, shown in yellow. Then they prepare fingerprint states $\left|s_{k}\right\rangle$ according to $E\left(x_{k}\right)$ and send them to a remote referee through the lossy channel of transmittance $\eta_{k}$. Finally, the referee can determine the relationship among $N$ messages, that is, output the relationship state $\mathcal{G}$.

\section{B. Protocol}

Here, we introduce our $N$-party quantum fingerprinting protocol, as illustrated in Fig. 1.

Protocol: $N$-party quantum fingerprinting protocol

Input: $N$ messages $\left\{x_{k}\right\}, k=1,2, \ldots, N$. Output: Relationship State $\mathcal{G}$.

\section{Preparation.}

$N$ senders $\left\{S_{k}\right\}$ use the error correction code (ECC) $E\left(x_{k}\right) \in\{0,1\}^{m}$ to encode messages $x_{k}$, where $m=\frac{n}{c}$ is the length of the $E\left(x_{k}\right), c<1$.

Remark 1: Since ECC is tolerable to errors up to a certain upper-bound, we shall regard the relationship of two $E\left(x_{k}\right)$ s to be different if the relative Hamming distance is larger than $\delta$ under which ECC runs successfully.

A table of the corresponding comparison results for all possible relationship states needs to be listed. (They are listed in Table I for the specific case $N=4$.)

2. Encoding.

Based on $E\left(x_{k}\right)$, every sender encodes their own fingerprint state $\left|s_{k}\right\rangle$, i.e., a product state of $m$ coherent states:

$$
\left|s_{k}\right\rangle=\bigotimes_{j=1}^{m}|\beta\rangle_{j}, \quad|\beta\rangle_{j}=\left|(-1)^{E\left(x_{k}\right)_{j}} \frac{\alpha_{k}}{\sqrt{m}}\right\rangle .
$$

Here $|\beta\rangle_{j}$ is a coherent state of amplitude $(-1)^{E\left(x_{k}\right)_{j}} \frac{\alpha_{k}}{\sqrt{m}}$, and $E\left(x_{k}\right)_{j}$ represents the $j$ th component in the entire sequence $E\left(x_{k}\right)$. Then they send their fin- 
gerprint state to the remote referee through the lossy channel of transmittance $\eta_{k}$, as illustrated in Fig. 1.

\section{Measurement.}

The referee uses several BSs and $N$ detectors $\left\{\mathrm{D}_{k}\right\}$ to perform the interference measurement of $N$ fingerprint states and records the number of counts $\left\{C_{k}\right\}$ of each detector. Here, we use $C_{k}$ to denote the number of counts of detector $\mathrm{D}_{k}$.

Remark 2: The referee can adopt the extendable device or the optimal device [20], which requires a minimum number of BSs, and the key point of the device is to realize the multibeam interference. In this work, we mainly analyze the optimal design based on balanced BSs, as illustrated in Fig. 3.

\section{Comparison.}

The referee selects appropriate $(N-1)$ threshold counts $\left\{C_{k}^{\text {th }}\right\}, k=2,3, \ldots, N$. Then, for a certain detector $\mathrm{D}_{k}$, if $C_{k}<C_{k}^{\mathrm{th}}$, the referee records the result as $R_{k}=0$, otherwise, records it as $R_{k}=1$. If the referee observes $N-1$ detectors, then he records it as $R_{2,3, \ldots, N}=R_{2} R_{3} \cdots R_{N}$.

Remark 3: Ideally, if $N$ messages $\left\{x_{k}\right\}$ are all the same, then only one detector $\mathrm{D}_{1}$ has clicks and if they are not all the same, then any detector $\mathrm{D}_{k}$ is likely to have clicks. However, in practice, due to the device imperfection such as the dark count probability of detectors, even $N$ messages are all the same, the detector $\mathrm{D}_{1}$ must have clicks and other detectors except $\mathrm{D}_{1}$ are also possible to click. Therefore, the referee needs to select the threshold $\left\{C_{k}^{\text {th }}\right\}, k=2,3, \ldots, N$. If $C_{k}<C_{k}^{\text {th }}$ holds from $k=2$ to $N$, then the referee concludes they are all the same; otherwise, he concludes they are not all the same.

\section{Decision.}

If the referee can get a complete relationship state $\mathcal{G}$, then he makes a decision of Yes and outputs $\mathcal{G}$.

Otherwise, the referee makes a decision of No. He changes the combination of the interference beams and returns to step 2 for next round.

The fingerprint state sent in round $t$ is

$$
\left|s_{k}^{t}\right\rangle=\bigotimes_{j=1}^{m}\left|\beta^{t}\right\rangle_{j}, \quad\left|\beta^{t}\right\rangle_{j}=\left|(-1)^{E\left(x_{k}\right)_{j}} \frac{\alpha_{k}^{t}}{\sqrt{m}}\right\rangle .
$$

The result of the round $t$ comparison can be recorded as $R_{2,3, \ldots, N}^{(t)}$, where $t=1,2, \ldots, T$ and $T$ represents the actual number of rounds.

Remark 4: It is possible that after the first round of steps $2-5$, the referee can only know $N$ messages are not all the same, but he still cannot determine the complete relationship state $\mathcal{G}$. So, the combinations of the interference beams need to be changed and steps 2-5 need to be run for several rounds until the referee can determine a unique $\mathcal{G}$ based on the comparison results.

We can use a flow chart to describe the protocol, as illustrated in Fig. 2.

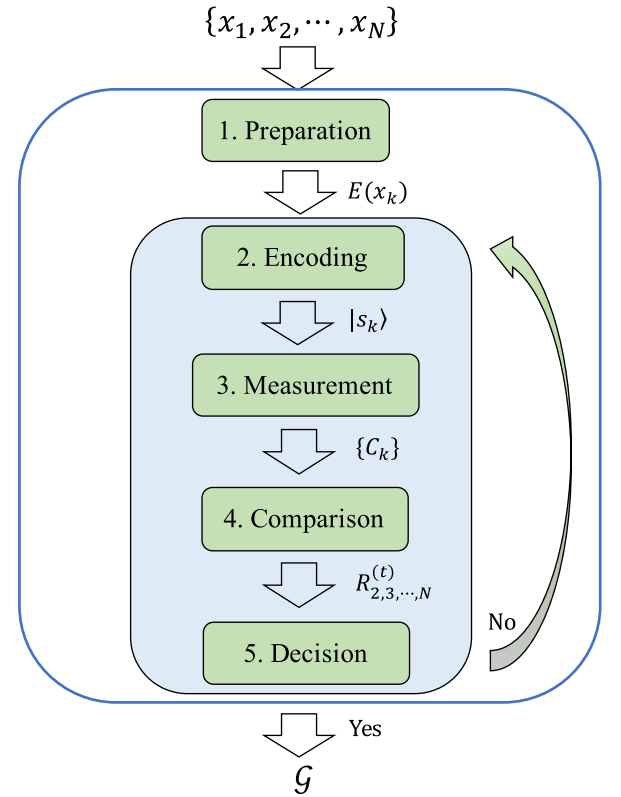

FIG. 2. The flow chart of $N$-party quantum fingerprinting protocol.

\section{The design of $N$-party quantum fingerprinting network based on balanced BSs}

We use the optimal device design that requires a minimum number of BSs [20]. When $N=2^{s}, s \in \mathbb{Z}^{+}$, we show in Fig. 3 how to determine $\mathcal{G}$ based on quantum fingerprinting network composed of balanced BSs. Each bracket, which represents for a balanced BS, compares its two inputs. The first layer shows the comparison results of $E\left(x_{k}\right)$ and $E\left(x_{k+1}\right)$, $k=1,3, \ldots, N-1$; the second layer shows the comparison results of $E\left(x_{k}\right)+E\left(x_{k+1}\right)$ and $E\left(x_{k+2}\right)+E\left(x_{k+3}\right), k=$ $1,5, \ldots, N-3$, and so on, and the sth layer shows the comparison results of $\sum_{k=1}^{2^{s-1}} E\left(x_{k}\right)$ and $\sum_{k=2^{s-1}+1}^{N} E\left(x_{k}\right)$.

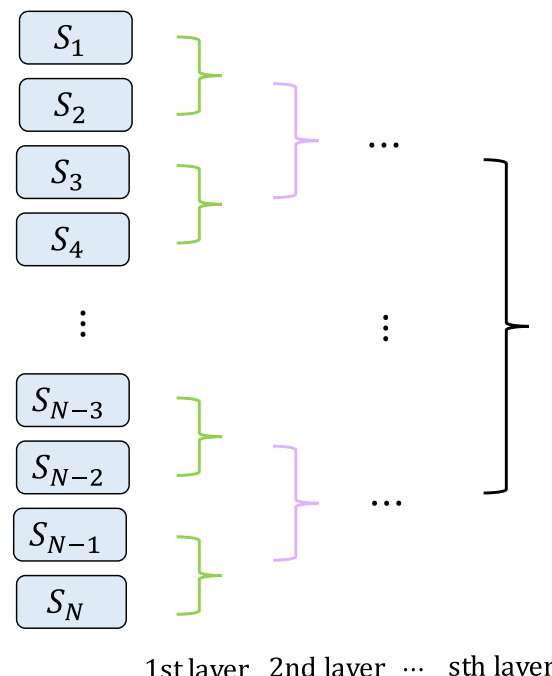

FIG. 3. The design of $N$-party quantum fingerprinting network based on balanced BSs, where $N=2^{s}, s \in \mathbb{Z}^{+} . N$ senders, $\left\{S_{k}\right\}$, hold the messages to be compared. Each bracket represents a balanced BS and compares the input of the two parts. The 1st, 2nd, and sth layer are shown in green, purple, and black, respectively. 

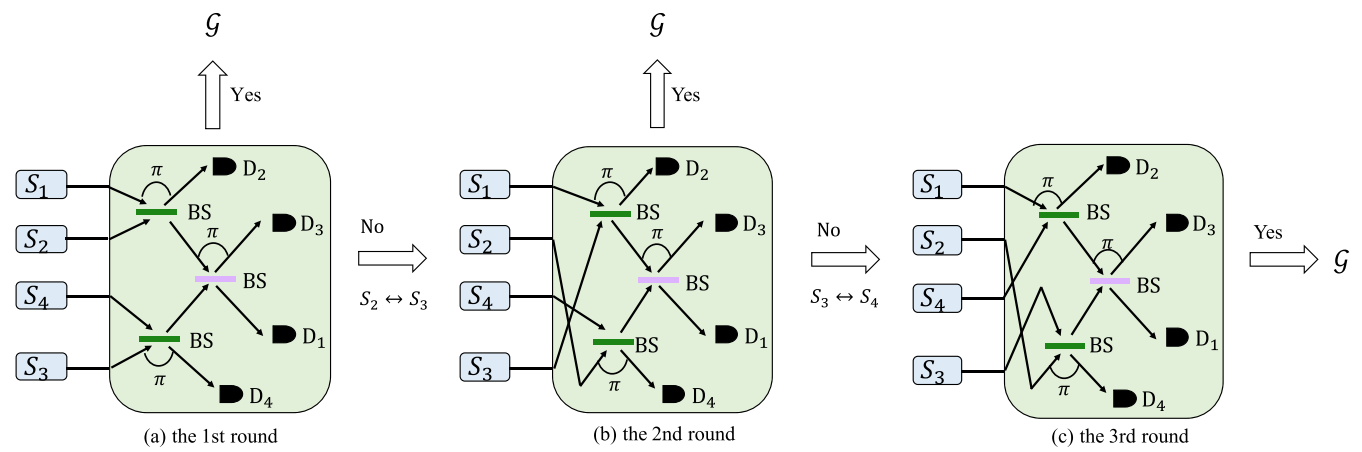

FIG. 4. Four-party quantum fingerprinting protocol based on balanced BSs. $\left\{S_{k}\right\}$ : four message-senders. BS: balanced beam-splitter. If the amplitudes of two incident states are equal, then the output light is supposed to be on the upper side (lower side) of the BS; if the amplitudes of two incident states are opposite, then the output light is supposed to be on the lower side (upper side) of the BS. The referee can know whether the corresponding messages are equal or not by observing the clicks of the corresponding detector. $\left\{\mathrm{D}_{k}\right\}$ : detectors. The referee observes the clicking results of $(N-1)$ detectors to determine the relationship state $\mathcal{G}$ of $T$ rounds.

When $N=4$, as shown in Fig. 4, the comparison results of the 1st layer are determined by the clicking results of detectors $\mathrm{D}_{2}$ and $\mathrm{D}_{4}$, and the comparison result of the 2nd layer is determined by that of detector $\mathrm{D}_{3}$. In the 1 st round, if $R_{2}=0$ $\left(R_{4}=0\right)$, then the two parts compared through $\mathrm{D}_{2}\left(\mathrm{D}_{4}\right)$ are equal, i.e., $E\left(x_{1}\right)=E\left(x_{2}\right)\left(E\left(x_{3}\right)=E\left(x_{4}\right)\right)$; if $R_{2}=1\left(R_{4}=\right.$ $1)$, then the two parts compared through $\mathrm{D}_{2}\left(\mathrm{D}_{4}\right)$ are different, i.e., $E\left(x_{1}\right) \neq E\left(x_{2}\right)\left(E\left(x_{3}\right) \neq E\left(x_{4}\right)\right)$; if $R_{3}=0\left(R_{3}=1\right)$, then the two parts compared through $\mathrm{D}_{3}$ are equal (different), i.e., $E\left(x_{1}\right)+E\left(x_{2}\right)=E\left(x_{3}\right)+E\left(x_{4}\right)\left(E\left(x_{1}\right)+E\left(x_{2}\right) \neq\right.$ $\left.E\left(x_{3}\right)+E\left(x_{4}\right)\right)$. Based on this, the error probability of 1 st round is

$$
\begin{gathered}
P_{e}^{(1)}=\max \left\{P_{2}^{\mathrm{E}}\left(R_{2}=1\right), P_{2}^{\mathrm{D}}\left(R_{2}=0\right),\right. \\
P_{3}^{\mathrm{E}}\left(R_{3}=1\right), P_{3}^{\mathrm{D}}\left(R_{3}=0\right), \\
\left.P_{4}^{\mathrm{E}}\left(R_{4}=1\right), P_{4}^{\mathrm{D}}\left(R_{4}=0\right)\right\},
\end{gathered}
$$

where $P_{k}^{r}\left(R_{k}\right)$ means the probability of the comparison result is $R_{k}$ when the two parts compared through $\mathrm{D}_{k}$ are equal ( $r=$ E) and different $(r=\mathrm{D})$. For example, $P_{2}^{\mathrm{E}}\left(R_{2}=1\right)$ means the probability of the comparison result is $R_{2}=1$ when $E\left(x_{1}\right)=$ $E\left(x_{2}\right)$.

The error probability of our protocol is

$$
P_{e}=\max \left\{P_{e}^{(t)}\right\}
$$

where $t=1,2, \ldots, T$, and $P_{e}^{(t)}$ represents the error probability of round $t$.

When $2^{s-1}<M<2^{s}=N$, the design in Fig. 3 can also be used to determine $\mathcal{G}$ for $M$ messages and we provide a detailed explanation in Appendix C. Therefore, our protocol can determine the relationship state $\mathcal{G}$ for any number of messages. In the following, we set $N=2^{s}, s \in \mathbb{Z}^{+}$if there is no special statement.

\section{Communication complexity}

If the referee only needs to judge whether $N$ messages are all the same $[20,21]$, then it is the special-case problem to judge whether $\mathcal{G}=\mathcal{G}^{\mathrm{AE}}$ in our protocol, with the total communication complexity of the classical optimal fingerprinting protocol $C_{\mathrm{o}}^{\mathrm{AE}}$ and the classical limit $C_{1}^{\mathrm{AE}}$ :

$$
\begin{aligned}
C_{\mathrm{o}}^{\mathrm{AE}}= & N\left[\frac{\log _{2}\left(P_{e}\right)}{\log _{2}\left[1-\frac{1}{9}\left(1-e^{-\frac{1}{2}}\right)\right]}\right] \\
\times & {\left[8 \sqrt{2\left\lceil\frac{3 n}{N}\right\rceil}+4\left[\log _{2}\left(3 n /\left\lceil\frac{3 n}{N}\right\rceil\right)\right],\right.} \\
& C_{1}^{\mathrm{AE}}=N\left[\frac{\left(1-2 \sqrt{P_{e}}\right) \sqrt{n}}{2 \sqrt{N \ln 2}}-\frac{1}{N}\right],
\end{aligned}
$$

where $P_{e}$ is the error probability of the classical fingerprinting protocol.

Completely determining the specific $\mathcal{G}$ is more difficult than just determining whether $\mathcal{G}=\mathcal{G}^{\mathrm{AE}}$. Obviously,

$$
C_{\mathrm{o}}^{\mathrm{R}}>C_{\mathrm{o}}^{\mathrm{AE}}, \quad C_{1}^{\mathrm{R}}>C_{1}^{\mathrm{AE}},
$$

where $C_{\mathrm{o}}^{\mathrm{R}}$ and $C_{1}^{\mathrm{R}}$ represent the optimal communication complexity in classical version and classical limit when determining $\mathcal{G}$, respectively.

In prior-art $N$-party quantum fingerprinting protocol [20], the referee only needs to judge whether $\mathcal{G}=\mathcal{G}^{\mathrm{AE}}$, the total communication complexity is

$$
Q^{\mathrm{AE}}=\sum_{k=1}^{N} \mu_{k} \log _{2} n,
$$

where $\mu_{k}=\left|\alpha_{k}\right|^{2}$ represents the total photon number of the fingerprint state in Eq. (3) sent by the $k$ th sender.

In our protocol, if the referee needs to run steps $2-5$ for $T$ rounds to determine a unique $\mathcal{G}$, the total communication complexity $Q^{\mathrm{R}}$ is

$$
Q^{\mathrm{R}}(T)=\sum_{t=1}^{T} \sum_{k=1}^{N} \mu_{k}^{t} \log _{2} n,
$$

where $\mu_{k}^{t}=\left|\alpha_{k}^{t}\right|^{2}$ represents the total photon number of the fingerprint state sent by the $k$ th sender of round $t$ of steps 2 5 in the protocol. We denote the largest possible number of rounds for steps $2-5$ as $\bar{T}(N)$ given $N$ messages, obviously, the upper bound of the communication complexity $Q^{\mathrm{R}}$ for our 
TABLE I. Determine $\mathcal{G}$ for four messages $\left\{x_{k}\right\}$ by three detectors $\mathrm{D}_{2}, \mathrm{D}_{3}$ and $\mathrm{D}_{4}$ in Fig. 4. $R_{2,3,4}^{(t)}$ represent the comparison results of the three detectors after round $t$ of the protocol. $\left[\widetilde{R_{2,3,4}^{(1)}}, \widetilde{R_{2,3,4}^{(2)}}, \widetilde{R_{2,3,4}^{(3)}}\right] \in$ $\{[101,111, \emptyset],[111,101, \varnothing],[111,111,101],[111,111,111]\}$, where $\emptyset$ means that there is no need for a third round.

\begin{tabular}{lccc}
\hline \hline Relationship State & $R_{2,3,4}^{(1)}$ & $R_{2,3,4}^{(2)}$ & $R_{2,3,4}^{(3)}$ \\
\hline $1\left\{\left\{x_{1}, x_{2}, x_{3}, x_{4}\right\}\right\}$ & 000 & & \\
$2\left\{\left\{x_{1}, x_{2}, x_{3}\right\},\left\{x_{4}\right\}\right\}$ & 011 & 011 & \\
$3\left\{\left\{x_{1}, x_{2}, x_{4}\right\},\left\{x_{3}\right\}\right\}$ & 011 & 110 & \\
$4\left\{\left\{x_{1}, x_{3}, x_{4}\right\},\left\{x_{2}\right\}\right\}$ & 110 & 011 & \\
$5\left\{\left\{x_{2}, x_{3}, x_{4}\right\},\left\{x_{1}\right\}\right\}$ & 110 & 110 & \\
$6\left\{\left\{x_{1}, x_{2}\right\},\left\{x_{3}, x_{4}\right\}\right\}$ & 010 & & \\
$7\left\{\left\{x_{1}, x_{3}\right\},\left\{x_{2}, x_{4}\right\}\right\}$ & 101 & 010 & \\
$8\left\{\left\{x_{1}, x_{4}\right\},\left\{x_{2}, x_{3}\right\}\right\}$ & 101 & 101 & \\
$9\left\{\left\{x_{1}, x_{2}\right\},\left\{x_{3}\right\},\left\{x_{4}\right\}\right\}$ & 011 & 111 & \\
$10\left\{\left\{x_{1}, x_{3}\right\},\left\{x_{2}\right\},\left\{x_{4}\right\}\right\}$ & 111 & 011 & \\
$11\left\{\left\{x_{1}, x_{4}\right\},\left\{x_{2}\right\},\left\{x_{3}\right\}\right\}$ & 111 & 111 & 011 \\
$12\left\{\left\{x_{2}, x_{3}\right\},\left\{x_{1}\right\},\left\{x_{4}\right\}\right\}$ & 111 & 111 & 110 \\
$13\left\{\left\{x_{2}, x_{4}\right\},\left\{x_{1}\right\},\left\{x_{3}\right\}\right\}$ & 111 & 110 & \\
$14\left\{\left\{x_{3}, x_{4}\right\},\left\{x_{1}\right\},\left\{x_{2}\right\}\right\}$ & 110 & 111 & $\widetilde{R_{2,3,4}^{(3)}}$ \\
$15\left\{\left\{x_{1}\right\},\left\{x_{2}\right\},\left\{x_{3}\right\},\left\{x_{4}\right\}\right\}$ & $\widetilde{R_{2,3,4}^{(1)}}$ & $\widetilde{R_{2,3,4}^{(2)}}$ & \\
\hline \hline
\end{tabular}

protocol is

$$
\overline{Q^{\mathrm{R}}}=\sum_{t=1}^{\bar{T}} \sum_{k=1}^{N} \mu_{k}^{t} \log _{2} n \geqslant Q^{\mathrm{R}}(T),
$$

where $Q^{\mathrm{R}}(T)$ is the actual communication complexity with $T$-round steps $2-5$.

To show the advantages over its classical version of our protocol in communication complexity, we compare $\overline{Q^{\mathrm{R}}}$ with $C_{\mathrm{o}}^{\mathrm{AE}}$ and $C_{1}^{\mathrm{AE}}$. Note that $\overline{Q^{\mathrm{R}}}$ is the upper bound of communication complexity of our protocol in Eq. (12), while $C_{\mathrm{o}}^{\mathrm{AE}}$ and $C_{1}^{\mathrm{AE}}$ are lower bounds of communication complexity of the classical versions in Eq. (9).

\section{RELATIONSHIP STATES OF FOUR-PARTY QUANTUM FINGERPRINTING PROTOCOL}

Next, we take the four-party quantum fingerprinting protocol through symmetric channel as an example to introduce our protocol in detail, as illustrated in Fig. 4.

\section{A. Relationship state $\mathcal{G}$ for four messages}

There are 15 possible relationship states for four messages $\left\{x_{k}\right\}$ in Table I. Since $\left\{x_{k}\right\}$ and $\left\{E\left(x_{k}\right)\right\}$ have the same relationship state, the referee only needs to know the relationship state of $\left\{E\left(x_{k}\right)\right\}$. But he needs neither $E\left(x_{k}\right)$ nor $x_{k}$ explicitly for the complete relationships.

As shown in Fig. 4 and Table I, on the first round, if the referee observes the any result in the 1st row or 6th row, he can determines the relationship state $\mathcal{G}$ completely.

Otherwise, he goes into the second round. If he observes any result in the range from 2 nd row to 5 th row, from 7 th row to 10th row, from 13th row to 14th row, or a result of $\{[101,111],[111,101]\}$ in 15 th row, then he can determine $\mathcal{G}$ completely.
TABLE II. Optimized parameters values of our four-party protocol. Here, $n=10^{13}, \delta=0.22, \eta=0.1, P_{d}=10^{-11}, c=0.2, \varepsilon=$ $10^{-2}$.

\begin{tabular}{lllll}
\hline \hline$\mu$ & $C_{2}^{\text {th }}$ & $C_{3}^{\text {th }}$ & $C_{4}^{\text {th }}$ & $\overline{Q^{\mathrm{R}}}$ \\
\hline 4961 & 602 & 553 & 602 & $2.57 \times 10^{6}$ \\
\hline
\end{tabular}

Otherwise, he goes into the third round to finalize the complete relationship state $\mathcal{G}$.

\section{B. Numerical results}

If the channel is symmetric, then we set the four senders' channel transmittance as $\eta$ and their average phonon number in every round as $\mu$ for our four-party protocol. We seek $\mu$ and $\left\{C_{k}^{\text {th }}\right\}, k=2,3,4$, to minimize $\overline{Q^{\mathrm{R}}}$ under the constraint $P_{e} \leqslant \varepsilon$, where $P_{e}$ is the error probability in Eq. (6).

For the calculation of error probability, we denote $\mathcal{C}_{k}^{r}$ as the random variable corresponding to the number of clicks in $\mathrm{D}_{k}$ for the cases $r$, where $r=\mathrm{E}$ or $r=\mathrm{D}$ means the two parts compared through $\mathrm{D}_{k}$ is equal or different, respectively. Notation $\mathcal{P}_{k}^{r}$ represents the probability of observing a click in each mode for the case of $r$ above through $\mathrm{D}_{k}$. For example, in our four-party protocol, $\mathcal{P}_{2}^{\mathrm{E}}$ represents the probability of observing a click in $\mathrm{D}_{2}$ conditional that each mode of $E\left(x_{1}\right)$ and $E\left(x_{2}\right)$ is equal.

In our four-party protocol,

$$
\begin{array}{ll}
\mathcal{P}_{2}^{\mathrm{E}}=P_{d}, & \mathcal{P}_{2}^{\mathrm{D}}=\delta\left(1-e^{-\frac{2 \eta \mu}{m}}\right)+P_{d}, \\
\mathcal{P}_{3}^{\mathrm{E}}=P_{d}, & \mathcal{P}_{3}^{\mathrm{D}}=\delta\left(1-e^{-\frac{\eta \mu}{m}}\right)+P_{d}, \\
\mathcal{P}_{4}^{\mathrm{E}}=P_{d}, & \mathcal{P}_{4}^{\mathrm{D}}=\delta\left(1-e^{-\frac{2 \eta \mu}{m}}\right)+P_{d},
\end{array}
$$

where $P_{d}$ represents the dark count probability of detectors and $\delta$ represents the smallest relative Hamming distance between any two distinct $E\left(x_{k}\right)$.

Once we know $\mathcal{P}_{k}^{\mathrm{E}}$ and $\mathcal{P}_{k}^{\mathrm{D}}$ of detector $\mathrm{D}_{k}$, we can choose an appropriate threshold $C_{k}^{\text {th }}$ to satisfy $P_{e} \leqslant \varepsilon$ :

$$
\begin{gathered}
P_{e}=\max \left\{P_{2}^{\mathrm{E}}\left(C_{2}>C_{2}^{\text {th }}\right), P_{2}^{\mathrm{D}}\left(C_{2}<C_{2}^{\text {th }}\right),\right. \\
P_{3}^{\mathrm{E}}\left(C_{3}>C_{3}^{\text {th }}\right), P_{3}^{\mathrm{D}}\left(C_{3}<C_{3}^{\text {th }}\right), \\
\left.P_{4}^{\mathrm{E}}\left(C_{4}>C_{4}^{\text {th }}\right), P_{4}^{\mathrm{D}}\left(C_{4}<C_{4}^{\text {th }}\right)\right\} .
\end{gathered}
$$

The optimized values of $\mu, C_{2}^{\text {th }}, C_{3}^{\text {th }}$, and $C_{4}^{\text {th }}$ are listed in Table II. With these parameters, we can calculate the upper bound of the communication complexity $\overline{Q^{\mathrm{R}}}=$ $2.57 \times 10^{6}$, while in the same condition, $C_{\mathrm{o}}^{\mathrm{AE}}=1.29 \times 10^{10}$, $C_{1}^{\mathrm{AE}}=3.04 \times 10^{6}$. This shows, given the same four messages, our protocol has an advantage by four magnitude orders in communication complexity reduction compared with the optimal classical version and breaks the classical limit.

\section{OPTIMIZATION OVER ASYMMETRIC CHANNEL}

Given an asymmetric channel, the optimization is more complicated, because there are more parameters to be optimized for minimization of $\overline{Q^{\mathrm{R}}}$. 
TABLE III. Optimized parameters values of the two-party quantum fingerprinting protocol through asymmetric channel. Here, $n=3 \times$ $10^{12}, c=0.2, P_{d}=10^{-10}, \delta=0.22, \sqrt{\eta_{1}}=0.3, \sqrt{\eta_{2}}=0.4, \varepsilon=10^{-5}$, for ideal BS, $v=1$, for realistic BS, $v=0.99$.

\begin{tabular}{|c|c|c|c|c|}
\hline & $\alpha_{1}$ & $\alpha_{2}$ & $C_{2}^{\text {th }}$ & $\overline{Q^{\mathrm{R}}}$ \\
\hline Our two-party protocol with ideal BS by one-bit method & 85 & 78 & 1685 & $5.52 \times 10^{5}$ \\
\hline Our two-party protocol with ideal BS by two-bit method & 69 & 70 & 898 & $3.91 \times 10^{5}$ \\
\hline Our two-party protocol with realistic BS by one-bit method & 88 & 77 & 1695 & $5.67 \times 10^{5}$ \\
\hline
\end{tabular}

\section{A. Two-party quantum fingerprinting protocol through asymmetric channel}

When $N=2$, we optimize $\alpha_{1}, \alpha_{2}$, and $C_{2}^{\text {th }}$ for the minimization of $\overline{Q^{\mathrm{R}}}$. The analytical expression of $\mathcal{P}_{k}^{r}$ can be found in Appendix A 1.

The optimized parameter values in the two-party protocol through asymmetric channel are listed in 2nd row of Table III. With these parameter values, we can calculate the upper bound of the communication complexity $\overline{Q^{\mathrm{R}}}=5.52 \times$ $10^{5}$. Compared with the communication complexity of the classical optimal version $C_{\mathrm{o}}^{\mathrm{AE}}=1.24 \times 10^{10}$ and the classical limit $C_{1}^{\mathrm{AE}}=1.46 \times 10^{6}$, our two-party protocol keeps its advantages of five-order of magnitude and breaks the classical limit.

\section{B. Fingerprinting network through asymmetric channel}

Here we study the optimization of four-party fingerprinting network through asymmetric channel. Detailed analytical expression of each $\mathcal{P}_{k}^{r}$ can be found in the Appendix A 2. In the asymmetric case, the fingerprint states sent by each sender in each round of steps 2-5 are always different from each other. To obtain the optimized result, the value of $\mathcal{P}_{k}^{r}$ is supposed to be different in different rounds, we use $\mathcal{P}_{k}^{r, t}$ to represent $\mathcal{P}_{k}^{r}$ in round $t$.

The optimized values of $\left\{\alpha_{k}^{t}\right\}$ and $\left\{C_{k}^{\text {th }}\right\}$ for different senders and in different rounds are listed in Table IV. We can calculate the upper bound of the communication complexity $\overline{Q^{\mathrm{R}}}=4.43 \times 10^{6}$, while in optimal classical protocol $C_{\mathrm{o}}^{\mathrm{AE}}=$ $1.01 \times 10^{11}$ and the classical limit is $C_{1}^{\mathrm{AE}}=1.19 \times 10^{7}$. Obviously, our four-party protocol through asymmetric channel shows an advantage by five orders of magnitude reduction in communication complexity compared with the optimal classical version. It also breaks the classical limit.

\section{DISCUSSION}

Given the same $N$ messages, the comparison of three multiparty fingerprinting protocols can be found in Table V. The

TABLE IV. Optimized parameter values of our four-party protocol through asymmetric channel. Here, $n=10^{14}, c=0.2, P_{d}=$ $10^{-11}, \delta=0.22, \sqrt{\eta_{1}}=0.3, \sqrt{\eta_{2}}=0.4, \sqrt{\eta_{3}}=0.5, \sqrt{\eta_{4}}=0.6$, $\varepsilon=10^{-5}$.

\begin{tabular}{lrrrrrrr}
\hline \hline Round & $\alpha_{1}$ & $\alpha_{2}$ & $\alpha_{3}$ & $\alpha_{4}$ & $C_{2}^{\text {th }}$ & $C_{3}^{\text {th }}$ & $C_{4}^{\text {th }}$ \\
\hline 1st & 109 & 109 & 69 & 69 & 5367 & 5700 & 5332 \\
2nd & 97 & 77 & 99 & 78 & 5519 & 5600 & 5439 \\
3rd & 90 & 84 & 85 & 91 & 5699 & 5600 & 5347 \\
\hline \hline
\end{tabular}

prior-art multiparty quantum fingerprinting protocol [20] can be regarded as the special case of our multiparty protocol, performing steps 2-5 only once and obtaining the partial relationship of whether $\mathcal{G}=\mathcal{G}^{\mathrm{AE}}$. In our protocol, by changing the combination of interference beams, the referee can determine all possible $\mathcal{G}$ for $N$ messages in $O(N)$ rounds. Compared with the prior-art multiparty quantum protocol [20], our protocol can provide complete relationships among all messages with only linear increase in communication complexity.

A complete result for $N$ parties in general is very hard, although we can have results for some special cases. When the number of messages $M$ satisfies $M=N=2^{s}, s \in \mathbb{Z}^{+}$, we can use the device based on balanced BSs, as shown in Fig. 3, and the largest possible number of rounds $\bar{T}=N-1$. When $2^{s-1}<M<2^{s}=N$, the device in Fig. 3 can also be used and we analyze this in detail in Appendix C.

To further improve the performance of the protocol, the senders can encode multiple bits of $E\left(x_{k}\right)$ in one $\left|\beta^{\prime}\right\rangle_{j}$. For example, we consider the following two-bit encoding method

$$
\begin{aligned}
\left|s_{k}\right\rangle & =\bigotimes_{j=1, \text { odd }}^{m}\left|\beta^{\prime}\right\rangle_{j}, \\
\left|\beta^{\prime}\right\rangle_{j} & =\left|l^{E\left(x_{k}\right)_{j} \oplus E\left(x_{k}\right)_{j+1}(-1)^{E\left(x_{k}\right)_{j}}} \frac{\alpha_{k}}{\sqrt{m / 2}}\right\rangle_{j},
\end{aligned}
$$

where $\imath^{2}=-1$ and $E\left(x_{k}\right)_{j} \oplus E\left(x_{k}\right)_{j+1}$ means $E\left(x_{k}\right)_{j}+$ $E\left(x_{k}\right)_{j+1} \bmod 2$.

For our one-bit encoding two-party protocol, the upper bound of the communication complexity $\overline{Q^{\mathrm{R}}}=5.52 \times 10^{5}$, while it is $3.91 \times 10^{5}$ in two-bit encoding two-party protocol as shown in Table III. Not only $\overline{Q^{\mathrm{R}}}$ in two-bit encoding protocol is smaller, but also the number of fingerprinting states sent by the senders is half that of the one-bit encoding method, saving the communication time. If the senders encode more bits in each coherent state, then it saves more communication time, but the technical difficulty in preparing the fingerprint state $\left|s_{k}\right\rangle$ may also increase. So in this work we only analyze one-bit and two-bit encoding method.

TABLE V. The comparison of three multiparty fingerprinting protocols. The symbol $\checkmark$ indicates that the corresponding relationship state can be determined. $Q$ stands for communication

\begin{tabular}{|c|c|c|}
\hline Protocol & $\mathcal{G}^{\mathrm{AE}} \mathcal{G}^{\mathrm{EE}} \mathcal{G}$ & $Q$ \\
\hline Multiparty classical protocol $[20,21]$ & $\checkmark$ & $O(\sqrt{n})$ \\
\hline Original multiparty quantum protocol [20] & $\checkmark$ & $O\left(\mu \log _{2} n\right)$ \\
\hline Our multiparty protocol & $\checkmark$ & $\checkmark O\left(\mu \log _{2} n\right)$ \\
\hline
\end{tabular}
complexity per sender per run. 
We analyze our protocol based on the ideal BSs with interference visibility $v=1$. The interference visibility of the realistic BS is not equal to 1 , for example, $v=0.99$ in Ref. [16]. We can extend our method with ideal BSs to that with realistic BSs. The imperfection of BSs only affects $\mathcal{P}_{k}^{r}$ as analyzed in the Appendix B. As shown in the last line of Table III, when taking this imperfection into consideration, $\overline{Q^{\mathrm{R}}}=5.67 \times 10^{5}$ increases a little, but still satisfies $\overline{Q^{\mathrm{R}}}<C_{1}^{\mathrm{AE}}<C_{\mathrm{o}}^{\mathrm{AE}}$, keeping the advantages of our protocol in communication complexity.

\section{CONCLUSION}

We propose the relationship state $\mathcal{G}$ to describe all possible relationships among $N$ messages. Based on this, we provide a general protocol of quantum fingerprinting network. We take the four-party quantum fingerprinting protocol as an example to analyze in detail how to determine $\mathcal{G}$ by changing the combination of interference beams. Compared with the optimal classical version, our protocol reduces the communication complexity exponentially. Different from prior-art multiparty quantum protocol, our protocol can provide complete relationships among all messages with only linear increase in communication complexity.

\section{ACKNOWLEDGMENTS}

We acknowledge the financial support in part by Ministry of Science and Technology of China through The National Key Research and Development Program of China Grant No. 2017YFA0303901 and National Natural Science Foundation of China Grants No. 11474182, No. 11774198, No. 11974204, and No. U1738142.

\section{APPENDIX A: OPTIMIZATION OF QUANTUM FINGERPRINTING PROTOCOL OVER ASYMMETRIC CHANNEL}

\section{Two-party quantum fingerprinting protocol through asymmetric channel}

When $N=2$, the referee has two detectors $\mathrm{D}_{1}$ and $\mathrm{D}_{2}$. Ideally, when $x_{1}=x_{2}$, only $\mathrm{D}_{1}$ has clicks; when $x_{1} \neq x_{2}$, both $\mathrm{D}_{1}$ and $\mathrm{D}_{2}$ have clicks. The probabilities of observing a click for the case of each mode of $E\left(x_{1}\right)$ and $E\left(x_{2}\right)$ are equal and different are

$$
\mathcal{P}_{2}^{\mathrm{E}}=\left[1-e^{-\frac{1}{2 m}\left(\sqrt{\eta_{1}} \alpha_{1}-\sqrt{\eta_{2}} \alpha_{2}\right)^{2}}\right]+P_{d}, \quad \mathcal{P}_{2}^{\mathrm{D}}=\delta\left[1-e^{-\frac{1}{2 m}\left(\sqrt{\eta_{1}} \alpha_{1}+\sqrt{\eta_{2}} \alpha_{2}\right)^{2}}\right]+(1-\delta)\left[1-e^{-\frac{1}{2 m}\left(\sqrt{\eta_{1}} \alpha_{1}-\sqrt{\eta_{2}} \alpha_{2}\right)^{2}}\right]+P_{d} .
$$

To further improve the performance of the protocol, we consider the two-bit encoding method. The following is the corresponding probability

$$
\begin{aligned}
& \mathcal{P}_{2}^{\mathrm{E}}=\left[1-e^{-\frac{1}{m}\left(\sqrt{\eta_{1}} \alpha_{1}-\sqrt{\eta_{2}} \alpha_{2}\right)^{2}}\right]+P_{d}, \\
& \mathcal{P}_{2}^{\mathrm{D}}=(1-\delta)^{2}\left[1-e^{-\frac{1}{m}\left(\sqrt{\eta_{1}} \alpha_{1}-\sqrt{\eta_{2}} \alpha_{2}\right)^{2}}\right]+2 \delta(1-\delta)\left[1-e^{-\frac{1}{m}\left(\left(\sqrt{\eta_{1}} \alpha_{1}\right)^{2}+\left(\sqrt{\eta_{2}} \alpha_{2}\right)^{2}\right)}\right]+\delta^{2}\left[1-e^{-\frac{1}{m}\left(\sqrt{\eta_{1}} \alpha_{1}+\sqrt{\eta_{2}} \alpha_{2}\right)^{2}}\right]+P_{d} .
\end{aligned}
$$

\section{Four-party quantum fingerprinting protocol through asymmetric channel}

Similar to Table I, the referee can determine $\mathcal{G}$ for four messages based on three detectors $\left\{\mathrm{D}_{k}\right\}, k=2,3,4$. The corresponding probability in round $t$ is

$$
\begin{aligned}
& \mathcal{P}_{2}^{\mathrm{E}, t}=\left[1-e^{-\frac{1}{2 m}\left(-\sqrt{\eta_{p}} \alpha_{p}^{t}+\sqrt{\eta_{q}} \alpha_{q}^{t}\right)^{2}}\right]+P_{d}, \quad \mathcal{P}_{2}^{\mathrm{D}, t}=\delta\left[1-e^{-\frac{1}{2 m}\left(\sqrt{\eta_{p}} \alpha_{p}^{t}+\sqrt{\eta_{q}} \alpha_{q}^{t}\right)^{2}}\right]+(1-\delta)\left[1-e^{-\frac{1}{2 m}\left(-\sqrt{\eta_{p}} \alpha_{p}^{t}+\sqrt{\eta_{q}} \alpha_{q}^{t}\right)^{2}}\right]+P_{d},
\end{aligned}
$$

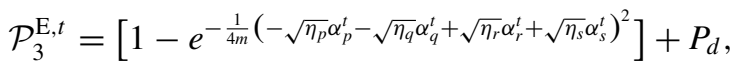

$$
\begin{aligned}
& \mathcal{P}_{3}^{\mathrm{D}, t}=\delta\left[1-e^{-\frac{1}{4 m}\left(x^{t}\right)^{2}}\right]+(1-\delta)\left[1-e^{-\frac{1}{4 m}\left(-\sqrt{\eta_{p}} \alpha_{p}^{t}-\sqrt{\eta_{q}} \alpha_{q}^{t}+\sqrt{\eta_{r}} \alpha_{r}^{t}+\sqrt{\eta_{s}} \alpha_{s}^{t}\right)^{2}}\right]+P_{d}, \\
& \mathcal{P}_{4}^{\mathrm{E}, t}=\left[1-e^{-\frac{1}{2 m}\left(-\sqrt{\eta_{r}} \alpha_{r}^{t}+\sqrt{\eta_{s}} \alpha_{s}^{t}\right)^{2}}\right]+P_{d}, \quad \mathcal{P}_{4}^{\mathrm{D}, t}=\delta\left[1-e^{-\frac{1}{2 m}\left(\sqrt{\eta_{r}} \alpha_{r}^{t}+\sqrt{\eta_{s}} \alpha_{s}^{t}\right)^{2}}\right]+(1-\delta)\left[1-e^{-\frac{1}{2 m}\left(-\sqrt{\eta_{r}} \alpha_{r}^{t}+\sqrt{\eta_{s}} \alpha_{s}^{t}\right)^{2}}\right]+P_{d},
\end{aligned}
$$

where $t=1,2,3$ and $\alpha_{k}^{t}$ represents the total amplitude of the fingerprint states sent by the $k$ th sender in round $t$ of steps 2-5; $(p, q)(r, s)=(1,2)(3,4),(1,3)(2,4),(1,4)(2,3)$ when $t=1,2,3$, respectively, and $x^{t}=\min \left\{\mid \sqrt{\eta_{p}} \alpha_{p}^{t}-\sqrt{\eta_{q}} \alpha_{q}^{t}+\sqrt{\eta_{r}} \alpha_{r}^{t}+\right.$ $\sqrt{\eta_{s}} \alpha_{s}^{t}||-,\sqrt{\eta_{p}} \alpha_{p}^{t}+\sqrt{\eta_{q}} \alpha_{q}^{t}+\sqrt{\eta_{r}} \alpha_{r}^{t}+\sqrt{\eta_{s}} \alpha_{s}^{t}||-,\sqrt{\eta_{p}} \alpha_{p}^{t}-\sqrt{\eta_{q}} \alpha_{q}^{t}-\sqrt{\eta_{r}} \alpha_{r}^{t}+\sqrt{\eta_{s}} \alpha_{s}^{t}||-,\sqrt{\eta_{p}} \alpha_{p}^{t}-\sqrt{\eta_{q}} \alpha_{q}^{t}+\sqrt{\eta_{r}} \alpha_{r}^{t}-$ $\left.\sqrt{\eta_{s}} \alpha_{s}^{t} \mid\right\}$. This means that $\alpha_{k}^{t}$ and $C_{k}^{\text {th }}$ in each run can de different with the purpose of minimizing $\overline{Q^{\mathrm{R}}}$ under the condition that $P_{e} \leqslant \varepsilon$.

\section{APPENDIX B: THE IMPACT OF INTERFERENCE VISIBILITY}

We can make some modifications to the probability of observing a click on detectors when considering the realistic BSs. Take the two-party quantum fingerprinting as an example. The corresponding probability is

$$
\begin{aligned}
\mathcal{P}_{2}^{\mathrm{E}}= & v\left[1-e^{-\frac{1}{2 m}\left(\sqrt{\eta_{1}} \alpha_{1}-\sqrt{\eta_{2}} \alpha_{2}\right)^{2}}\right]+(1-v)\left[1-e^{-\frac{1}{2 m}\left(\sqrt{\eta_{1}} \alpha_{1}+\sqrt{\eta_{2}} \alpha_{2}\right)^{2}}\right]+P_{d}, \\
\mathcal{P}_{2}^{\mathrm{D}}= & \delta\left[v\left(1-e^{-\frac{1}{2 m}\left(\sqrt{\eta_{1}} \alpha_{1}+\sqrt{\eta_{2}} \alpha_{2}\right)^{2}}\right)+(1-v)\left(1-e^{-\frac{1}{2 m}\left(\sqrt{\eta_{1}} \alpha_{1}-\sqrt{\eta_{2}} \alpha_{2}\right)^{2}}\right)\right]+(1-\delta)\left[v\left(1-e^{-\frac{1}{2 m}\left(\sqrt{\eta_{1}} \alpha_{1}-\sqrt{\eta_{2}} \alpha_{2}\right)^{2}}\right)\right. \\
& \left.+(1-v)\left(1-e^{-\frac{1}{2 m}\left(\sqrt{\eta_{1}} \alpha_{1}+\sqrt{\eta_{2}} \alpha_{2}\right)^{2}}\right)\right]+P_{d} .
\end{aligned}
$$

Obviously, when $v=1$, the expressions above becomes the probabilities in the ideal case, as shown in Eq. (A1). 
As shown in Table III, when taking this imperfection into consideration, $\overline{Q^{\mathrm{R}}}$ increases a little, but still satisfies $\overline{Q^{\mathrm{R}}}<C_{1}^{\mathrm{AE}}<$ $C_{\mathrm{o}}^{\mathrm{AE}}$, keeping the advantages in communication complexity of our protocol.

The analysis in the multiparty quantum fingerprinting protocol is similar.

\section{APPENDIX C: DETERMINE $\mathcal{G}$ FOR $M$ MESSAGES WITH THE DEVICE OF N-PARTY QUANTUM FINGERPRINTING PROTOCOL}

The device composed of balanced BSs, which can be used for $N$-party quantum fingerprinting, $N=2^{s}, s \in \mathbb{Z}^{+}$, can also be used to determine $\mathcal{G}$ for $M$ messages $(M<N)$. We show how to determine $\mathcal{G}$ for three messages using the four-party device in Fig. 4.

For example, when there are three messages for comparison, the sender $S_{1}$ prepares two fingerprint states $\left|s_{1}\right\rangle$, then sends them to the remote referee. The referee combines $\left|s_{1}\right\rangle$ with $\left|s_{2}\right\rangle$ and $\left|s_{1}\right\rangle$ with $\left|s_{3}\right\rangle$ for the interference measurement. He can determine $\mathcal{G}$ of three messages according to $R_{2,3,4}^{(1)}$, as shown in Table VI.

The referee needs to change combination of interference beams to determine $\mathcal{G}$ in three-party quantum fingerprinting
TABLE VI. Determine $\mathcal{G}$ for three messages $\left\{x_{1}, x_{2}, x_{3}\right\}$ by detectors $\mathrm{D}_{2}, \mathrm{D}_{3}$, and $\mathrm{D}_{4}$ in Fig. 4 .

\begin{tabular}{cc}
\hline \hline Relationship state & $R_{2,3,4}^{(1)}$ \\
\hline$\left\{\left\{x_{1}, x_{2}, x_{3}\right\}\right\}$ & 000 \\
$\left\{\left\{x_{1}, x_{2}\right\},\left\{x_{3}\right\}\right\}$ & 011 \\
$\left\{\left\{x_{1}, x_{3}\right\},\left\{x_{2}\right\}\right\}$ & 110 \\
$\left\{\left\{x_{2}, x_{3}\right\},\left\{x_{1}\right\}\right\}$ & 101 \\
$\left\{\left\{x_{1}\right\},\left\{x_{2}\right\},\left\{x_{3}\right\}\right\}$ & 111 \\
\hline \hline
\end{tabular}

based on extendable design [20], which can be completed for three rounds at most. Our method above can determine $\mathcal{G}$ in one round and does not require unbalanced BSs, which is very beneficial for experiments.
[1] C. H. Bennett and G. Brassard, Quantum cryptography: Public key distribution and coin tossing, in Proceedings of the IEEE International Conference on Computers, Systems, and Signal Processing (IEEE, Piscataway, NJ, 1984), pp. 175-179.

[2] N. Gisin, G. G. Ribordy, W. Tittel, and H. Zbinden, Quantum cryptography, Rev. Mod. Phys. 74, 145 (2002).

[3] X.-B. Wang, T. Hiroshima, A. Tomita, and M. Hayashi, Quantum information with Gaussian states, Phys. Rep. 448, 1 (2007).

[4] N. Gisin and R. Thew, Quantum communication, Nat. Photon. 1, 165 (2007).

[5] V. Scarani, H. Bechmann-Pasquinucci, N. J. Cerf, M. Dusek, N. Lutkenhaus, and M. Peev, The security of practical quantum key distribution, Rev. Mod. Phys. 81, 1301 (2009).

[6] S. Pirandola, U. L. Andersen, L. Banchi, M. Berta, D. Bunandar, R. Colbeck, D. Englund, T. Gehring, C. Lupo, and C. Ottaviani, Advances in quantum cryptography, Adv. Opt. Photon. 12, 1012 (2020).

[7] F. Xu, X. Ma, Q. Zhang, H.-K. Lo, and J.-W. Pan, Secure quantum key distribution with realistic devices, Rev. Mod. Phys. 92, 025002 (2020).

[8] H. Buhrman, R. Cleve, S. Massar, and R. deWolf, Nonlocality and communication complexity, Rev. Mod. Phys. 82, 665 (2010).

[9] H. Buhrman, W. vanDam, P. Hoyer, and A. Tapp, Multiparty quantum communication complexity, Phys. Rev. A 60, 2737 (1999).

[10] I. Newman and M. Szegedy, Public vs. private coin flips in one round communication games, in Proceedings of the 28th Annual ACM Symposium on Theory of Computing (ACM, Philadelphia, United States, 1996), pp. 561-570.

[11] L. Babai and P. G. Kimmel, Randomized simultaneous messages: Solution of a problem of Yao in communication complexity, in Proceedings of the 12th Annual IEEE Conference on Computational Complexity (Formerly: Structure in Complex- ity Theory Conference) (Cat. No. 97CB36072) (IEEE Computer Society, USA, 1997), pp. 239-246.

[12] H. Buhrman, R. Cleve, J. Watrous, and R. de Wolf, Quantum fingerprinting, Phys. Rev. Lett. 87, 167902 (2001).

[13] S. Massar, Quantum fingerprinting with a single particle, Phys. Rev. A 71, 012310 (2005).

[14] A. C.-C. Yao, Some complexity questions related to distributive computing (preliminary report), in Proceedings of the Eleventh Annual ACM Symposium on Theory of Computing (ACM, New York, NY, USA, 1979), pp. 209-213.

[15] J. M. Arrazola and N. Lutkenhaus, Quantum fingerprinting with coherent states and a constant mean number of photons, Phys. Rev. A 89, 062305 (2014).

[16] F. H. Xu, J. M. Arrazola, K. Wei, W. Wang, P. Palaciosavila, C. Feng, S. Sajeed, N. Lutkenhaus, and H. Lo, Experimental quantum fingerprinting with weak coherent pulses, Nat. Commun. 6, 8735 (2015).

[17] J.-Y. Guan, F. Xu, H.-L. Yin, Y. Li, W.-J. Zhang, S.-J. Chen, X.-Y. Yang, L. Li, L. X. You, T. Y. Chen, Z. Wang, Q. Zhang, and J. W. Pan, Observation of quantum fingerprinting beating the classical limit. Phys. Rev. Lett. 116, 240502 (2016).

[18] B. Lovitz and N. Lutkenhaus, Families of quantum fingerprinting protocols, Phys. Rev. A 97, 032340 (2018).

[19] X. Zhong, F. Xu, H.-K. Lo, and L. Qian, Efficient experimental quantum fingerprinting with wavelength division multiplexing, arXiv:2005.06049 (2020).

[20] H. Gómez-Sousa, Multiparty quantum fingerprinting with weak coherent pulses: Circuit design and protocol analysis, New J. Phys. 22, 113004 (2020).

[21] O. Fischer, R. Oshman, and U. Zwick, Public vs. private randomness in simultaneous multiparty communication complexity, in Proceedings of the 23rd International Colloquium on Structural Information and Communication Complexity (SIROCCO'16), Helsinki, Finland, July 19-21 (Springer International Publishing, Cham, 2016), pp. 60-74. 